\title{
A COMMUNITY OF CLONES: SNOW ALGAE ARE DIVERSE COMMUNITIES OF SPATIALLY STRUCTURED CLONES
}

\author{
Shawn P. Brown, ${ }^{1, *}$ Mark C. Ungerer,* and Ari Jumpponen* \\ *Division of Biology, Kansas State University, Manhattan, Kansas 66506, USA \\ Editor: Linda E. Graham
}

\begin{abstract}
Premise of research. Snow algae are cosmopolitan and often colonize late-season snowpacks. These snow algae do not occur in isolation; rather, visible algal blooms consist of multispecies communities. Although several of these common snow algae have been characterized taxonomically, their inter- and intraspecific diversity remains unknown. Further, the phylogeographic and biogeographic structuring of snow algal species is poorly understood.
\end{abstract}

Methodology. Algal communities were censused by sequencing the variable internal transcribed spacer 2 locus using Illumina MiSeq. We further analyzed two of the most common and abundant algal operational taxonomic units (OTUs) for biogeographic haplotype diversity.

Pivotal results. Our data show that the communities are diverse and taxonomically broad (orders: Chlamydomonadales [ $74 \%$ of OTUs], Microthamniales [20\% OTUs], and Chlorellales [ $6 \%$ OTUs]). We demonstrate that the two most common species (best nucleotide basic local alignment search tool match to Coenochloris sp. and Chlamydomonas sp.) have distinct haplotype distributions locally and regionally. Each sampled algal colony was dominated by one and only one haplotype, with negligible intraspecific haplotype diversity.

Conclusions. Our results suggest that snow algae are communities of clones within a discrete patch yet are heterogeneous across the landscape. Thus, these communities are likely structured via strong priority effects, intense kin competition, and dispersal limitations.

Keywords: snow algae, haplotype, biogeography, clonal, Illumina MiSeq.

Online enhancements: appendix tables and figures, data files.

\section{Introduction}

Snowpacks that persist into the alpine spring and summer are often colonized by algae. These algal blooms can manifest as visually striking colonization patches consisting of numerous algal cells. Often, these patches have distinct boundaries separating colonized snows from noncolonized snows (Brown et al. 2015a). Frequently, these snow algal blooms are visually red but can also be green or yellow depending on which algal species dominates the community. Red-pigmented algal blooms result from the enormous cell density of primarily Chlamydomonas nivalis or Chloromonas nivalis, whose red color is a result of astaxanthin (Müller et al. 1998), a secondary carotenoid, and its fatty acid ester derivatives (Gorton et al. 2001).

In combined snow and ice algal blooms, cell counts may reach as high as $\sim 500,000$ cells $\mathrm{mL}^{-1}$ (Yoshimura et al. 1997),

\footnotetext{
${ }^{1}$ Author for correspondence; current address: Department of Plant Biology, University of Illinois at Urbana-Champaign, Urbana, Illinois 61801, USA; e-mail: spbrown1@illinois.edu.
}

Manuscript received November 2015; revised manuscript received January 2016; electronically published April 21, 2016. but cell abundances within red-pigmented algal blooms solely within a snow matrix may be much lower $\left(\sim 5000\right.$ cells $\mathrm{mL}^{-1}$; Fogg 1967). Although these algae-colonized snows can harbor diverse communities of algae (Fujii et al. 2010; Remias et al. 2010; Lutz et al. 2015), the importance of the spatial structuring of these communities remains unknown. Previous queries into the ecology of snow algae have been limited mainly to community composition (Yoshimura et al. 1997; Takeuchi 2013). The increasing use of DNA meta-bar-coding tools has elucidated the hidden diversity of algae and presents an opportunity to integrate these new discoveries with traditional taxonomy (De Clerck et al. 2013). Snow algae have been described as simple communities that consist of few species (Takeuchi 2001) and are generally considered to occur in closed ecosystems (Hoham and Duval 2001). Most biogeographic studies of snow algae focus on altitudinal gradients and demonstrate that these simple communities drastically differ in the altitudinal patterning of their cell abundances (Yoshimura et al. 1997; Takeuchi 2001; Takeuchi and Kohshima 2004), suggesting that snow algae are strongly ordered by differences in ice pack structure even at a local scale. It is uncertain whether the observed differences between these snow algae result from geographic distance, differences in substrate quality, or subtle meteorolog- 
ical or topological differences. Additionally, to the best of our knowledge, investigations into the biogeography of snow algae using locus-targeted sequencing integrating community ecology and phylogenetic structure are few. In one of the few studies to investigate broad algal biogeographic patterns, Lutz et al. (2015) found that disparate sampling locations often consist of distinct algal communities, but individual algal taxon responses were not queried.

We aimed to investigate the spatial and temporal dynamics of snow algae communities. Additionally, we explored the intraspecific heterogeneity of the two most common snow algae across regions and years. We used a haplotype analysis framework to ask questions about the biogeographic structuring of snow algae populations from regional to local scales. Our results provide new insights into the distribution of snow algae and address questions about the microevolutionary forces that drive colonization by these organisms of adjacent but discrete patches of snow.

\section{Material and Methods}

\section{Sampling Locations and Procedures}

Algae-colonized snows were collected over 2 years (2011 and 2012) at sites in the states of Colorado (Niwot Ridge LongTerm Ecological Research site [2011] and Indian Peaks Wilderness area within the Arapaho and Roosevelt National Forest [2012]) and Washington (Glacier Peak Wilderness Area within the Wenatchee National Forest [2011, 2012]; see table 1; fig. 1). All accessible snows with algal colonization were sampled; many snows with apparent colonization were inaccessible (similar to Müller et al 1998). All samples were in full sun conditions and in the open landscape. Algae-colonized snows were sampled by taking five $\sim 85 \mathrm{~cm}^{3}$ surface $(\sim 5 \mathrm{~cm}$ depth) subsamples within visibly algal blooms from an area of about $2 \mathrm{~m}^{2}$ from each colonized snow patch. Surface snow was targeted here because snows nearer the surface have more algal cells compared to deeper snows (Grinde 1983), presumably due to higher photosynthetic capabilities with more direct sunlight. Snows with visible anthropogenic interference were avoided. Collected snows were allowed to melt under ambient conditions and homogenized, and $100 \mathrm{~mL}$ of the meltwater was filtered onto 2- $\mu \mathrm{m}$ Nucleopore Track-Etch Membrane filters (Whatman, Kent).

\section{Sequencing and Bioinformatics}

The Nucleopore filters were placed into MoBio UltraClean Soil DNA Isolation Kit (Carlsbad, CA) bead tubes. Total genomic DNA was extracted according to the manufacturer's protocol with the following modifications: (1) the filters were sonicated in the bead tubes for $10 \mathrm{~min}$ to dislodge adhered particles, and (2) two 2.4-mm zirconia beads (Bio-Spec, Bartlesville, OK) were added and homogenized in a FastPrep instrument (FP120, ThermoFisher Scientific, Waltham, MA) at a setting of 4.0 for $60 \mathrm{~s}$ to ensure complete lysis of cells. A locus-targeted amplicon library was generated in $50 \mu \mathrm{L}$ polymerase chain reactions (PCRs) using the following: $1 \mu \mathrm{M}$ forward and reverse primers (specific to the internal transcribed spacer 2 [ITS2] region of the rRNA gene repeat [fITS7; Ihrmark et al. 2012] and ITS4 [White et al. 1990]), sample-specific 12 base pair (bp) multiplex identification tags (MIDs) synthesized along the reverse ITS4 primer for a final reverse primer construct of MID-ITS4 (see table A1 for primer and MID sequences; tables A1-A3 available online), $10 \mathrm{ng}$ template DNA, $200 \mu \mathrm{M}$ of each deoxynucleotide, $2.5 \mathrm{mM} \mathrm{MgCl}_{2}, 10 \mu \mathrm{L} 5 \mathrm{X}$ Green GoTaq Flexi Buffer (Promega, Madison, WI), 14.6 $\mu \mathrm{L}$ molecular biologygrade water, and 2 U GoTaq Hot Start Polymerase (Promega, Madison, WI). PCR cycle parameters consisted of a $94^{\circ} \mathrm{C}$ initial denaturing step for $4 \mathrm{~min}$, followed by 30 cycles of $94^{\circ} \mathrm{C}$ for $1 \mathrm{~min}, 54^{\circ} \mathrm{C}$ annealing for $1 \mathrm{~min}$, and a $72^{\circ} \mathrm{C}$ extension step for $2 \mathrm{~min}$, followed by a final extension step at $72^{\circ} \mathrm{C}$ for $10 \mathrm{~min}$. Illumina sequencing linkers were ligated onto the amplicon constructs, and the amplicon library was sequenced on par-

Table 1

Sampling Locations of the Snow Algae for 2011 and 2012

\begin{tabular}{|c|c|c|c|c|c|c|c|}
\hline Year & State & Site & Landmark & Latitude & Longitude & Date & Elevation $(\mathrm{m})$ \\
\hline 2011 & Colorado & Niwot 1 & Near Soddie Laboratory & $40^{\circ} 02^{\prime} 56^{\prime \prime} \mathrm{N}$ & $105^{\circ} 34^{\prime} 51^{\prime \prime} \mathrm{W}$ & Aug. 10 & 3368 \\
\hline 2011 & Colorado & Niwot 2 & Saddle & $40^{\circ} 03^{\prime} 30^{\prime \prime} \mathrm{N}$ & $105^{\circ} 35^{\prime} 20^{\prime \prime} \mathrm{W}$ & Aug. 10 & 3514 \\
\hline 2011 & Washington & Lyman 1 & Cloudy Pass & $48^{\circ} 12^{\prime} 09^{\prime \prime} \mathrm{N}$ & $120^{\circ} 55^{\prime} 28^{\prime \prime} \mathrm{W}$ & Sept. 13 & 1961 \\
\hline 2011 & Washington & Lyman 2 & Terminal Moraine & $48^{\circ} 10^{\prime} 59^{\prime \prime} \mathrm{N}$ & $120^{\circ} 54^{\prime} 11^{\prime \prime} \mathrm{W}$ & Sept. 13 & 1802 \\
\hline 2011 & Washington & Lyman 3 & Lyman Glacier & $48^{\circ} 10^{\prime} 21^{\prime \prime} \mathrm{N}$ & $120^{\circ} 53^{\prime} 50^{\prime \prime} \mathrm{W}$ & Sept. 14 & 1880 \\
\hline 2011 & Washington & Lyman 4 & Spider Gap N & $48^{\circ} 10^{\prime} 14^{\prime \prime} \mathrm{N}$ & $120^{\circ} 52^{\prime} 55^{\prime \prime} \mathrm{W}$ & Sept. 14 & 2135 \\
\hline 2011 & Washington & Lyman 5 & Spider Gap S & $48^{\circ} 10^{\prime} 10^{\prime \prime} \mathrm{N}$ & $120^{\circ} 52^{\prime} 53^{\prime \prime} \mathrm{W}$ & Sept. 14 & 2123 \\
\hline 2011 & Washington & Lyman 6 & Lower Spider Snowfield & $48^{\circ} 09^{\prime} 42^{\prime \prime} \mathrm{N}$ & $120^{\circ} 52^{\prime} 42^{\prime \prime} \mathrm{W}$ & Sept. 14 & 1897 \\
\hline 2012 & Colorado & Indian Peaks 1 & E of Shoshoni Peak & $40^{\circ} 04^{\prime} 02^{\prime \prime} \mathrm{N}$ & $105^{\circ} 37^{\prime} 44^{\prime \prime} \mathrm{W}$ & July 15 & 3407 \\
\hline 2012 & Colorado & Indian Peaks 2 & S Shore Lake Isabelle & $40^{\circ} 04^{\prime} 00^{\prime \prime} \mathrm{N}$ & $105^{\circ} 04^{\prime} 01^{\prime \prime} \mathrm{W}$ & July 15 & 3358 \\
\hline 2012 & Washington & Lyman 1 & Cloudy Pass & $48^{\circ} 12^{\prime} 10^{\prime \prime} \mathrm{N}$ & $120^{\circ} 55^{\prime} 27^{\prime \prime} \mathrm{W}$ & Aug. 2 & 1966 \\
\hline 2012 & Washington & Lyman 2 & Terminal Moraine & $48^{\circ} 10^{\prime} 58^{\prime \prime} \mathrm{N}$ & $120^{\circ} 54^{\prime} 11^{\prime \prime} \mathrm{W}$ & Aug. 2 & 1794 \\
\hline 2012 & Washington & Lyman 3 & Lyman Glacier & $48^{\circ} 10^{\prime} 24^{\prime \prime} \mathrm{N}$ & $120^{\circ} 53^{\prime} 49^{\prime \prime} \mathrm{W}$ & Aug. 2 & 1866 \\
\hline 2012 & Washington & Lyman 4 & Spider Gap N & $48^{\circ} 10^{\prime} 14^{\prime \prime} \mathrm{N}$ & $120^{\circ} 52^{\prime} 55^{\prime \prime} \mathrm{W}$ & Aug. 2 & 2173 \\
\hline 2012 & Washington & Lyman 5 & Spider Gap S & $48^{\circ} 10^{\prime} 10^{\prime \prime} \mathrm{N}$ & $120^{\circ} 52^{\prime} 53^{\prime \prime} \mathrm{W}$ & Aug. 2 & 2137 \\
\hline 2012 & Washington & Lyman 6 & Lower Spider Snowfield & $48^{\circ} 09^{\prime} 41^{\prime \prime} \mathrm{N}$ & $120^{\circ} 52^{\prime} 35^{\prime \prime} \mathrm{W}$ & Aug. 2 & 1893 \\
\hline
\end{tabular}

Note. $\quad$ Niwot $=$ Niwot Ridge Long-Term Ecological Research site, Colorado; Indian Peaks = Indian Peaks Wilderness area, Colorado; Lyman = Lyman Glacier basin within the Glacier Peak Wilderness area, Washington. 


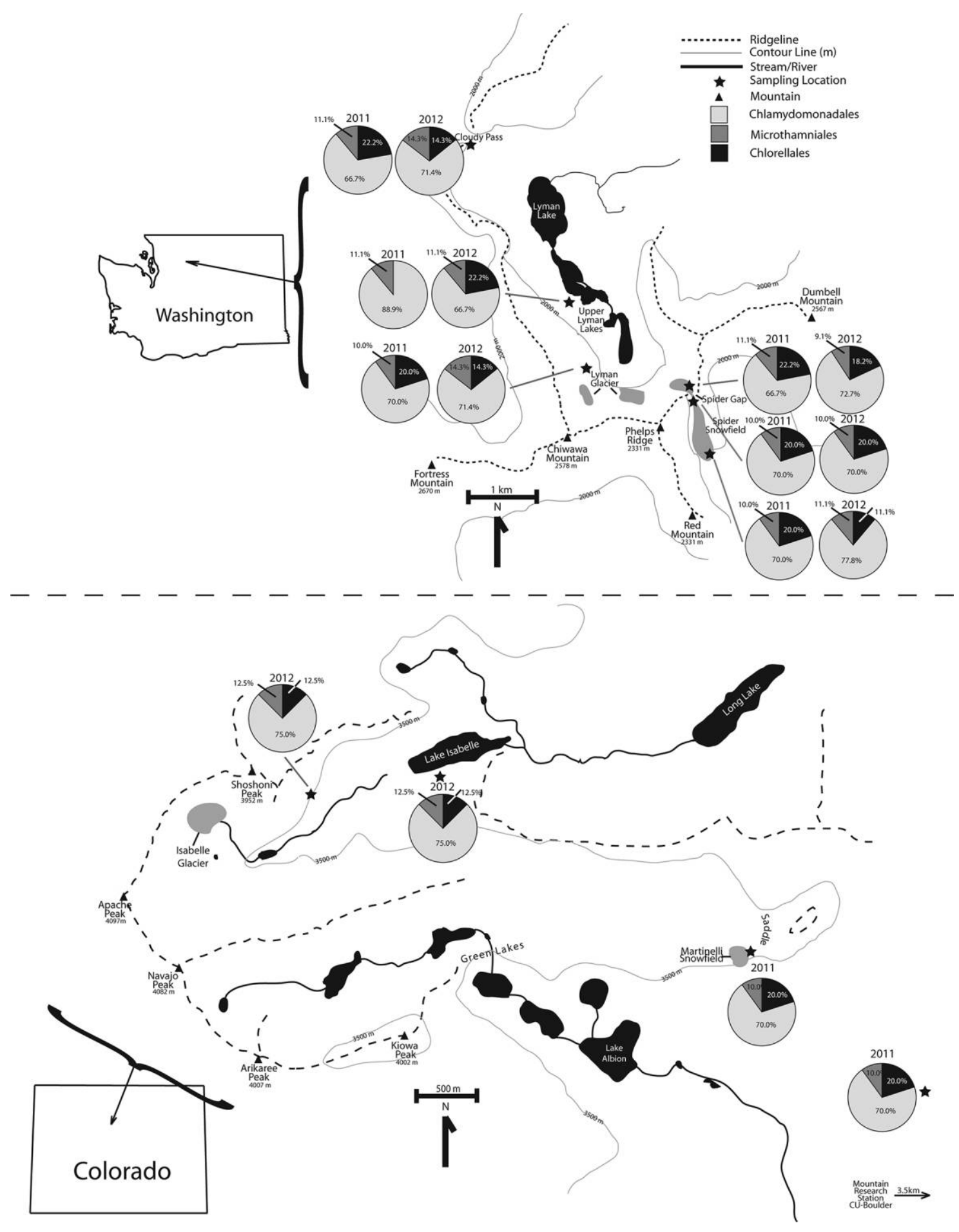

Fig. 1 Map of sampling locations (stars) in Washington (top) and Colorado (bottom) with other relevant geographic landmarks. The pie charts represent snow algae operational taxonomic unit (OTU) abundances on an order level for 2011 and 2012. OTUs were widely spread and found in multiple years and across a semicontinental scale. For each sampling location, haplotype proportions are reported in table A2.

tial Illumina MiSeq $(2 \times 250)$ reaction at the Kansas State University Integrated Genomics Facility (Manhattan, KS).

After stringent raw-sequence quality control and cleanup (see Brown et al. 2015a), a total of 369,651 algal sequences remained. These sequences were clustered into operational taxonomic units (OTUs) at a $97 \%$ similarity threshold using the average-neighbor algorithm (unweighted pair group method with arithmetic mean). All sequence quality control and clus- 
tering was done using the program Mothur (ver. 1.31.1; Schloss et al. 2009). To eliminate the potential inclusion of OTU artifacts due to inclusion of rare OTUs (Brown et al 2015b), only OTUs that consisted of more than 100 sequences were retained for analysis (the retained OTUs represented $\geq 98 \%$ of all algal sequences). The two most abundant OTUs by sequence count had close affinities (based on nucleotide basic local alignment search tool [BLASTn] queries against GenBank) to two snow algae, Coenochloris sp. and Chlamydomonas sp., both of which were abundant and dominated across all sampling sites. The OTU with affinity to Chlamydomonas sp. likely represents Chlamydomonas nivalis (Bau.) Wille (division: Chlorophyta; Gradinger and Nürnberd 1996), a red-pigmented chlorophyte that visually dominates in red snow. We refer to this OTU as Chlamydomonas sp. from this point forward, because we cannot unambiguously place it to C. nivalis as no representative ITS2 sequences for this taxon existed in the combined global nucleotide databases at the time of this analysis. The identity of the second OTU with the best BLASTn affinity to Coenochloris is even less clear. There is a dearth of genetic information about these snow algae, and their taxonomic identities remain unresolved. Notwithstanding, we hereafter refer to this alga as Coenochloris sp.

\section{Haplotype Analyses}

Since these two OTUs were highly abundant and widely distributed, we deemed them sufficiently abundant for population analyses. All sequences belonging to these two OTUs were harvested from the FASTA file after contig generation. Given the highly skewed OTU abundance distribution across all locations $(n=16)$, we randomly selected 50 sequences without replacement from each sampling location for each of the two OTUs (total of 800 sequences each for Coenochloris and Chlamydomonas; FASTA files provided in the supplemental files, available online). We tested whether deeper subsampling would generate additional haplotype diversity using Boneh estimates (Boneh et al. 1998) to predict the number of additional observed haplotypes in sampling schemes doubling our sampling depth; none of our samples included additional complete haplotypes in these analyses. As a result, we concluded that our subsampling depth was sufficient to capture the majority of haplotype diversity (see table A3).

Using the program Mothur, the 800 con-OTU (conspecific) sequences for each alga (50 from each sampling location) were truncated to $325 \mathrm{bp}$ after removal of primers and MIDs. The truncated sequences were pairwise aligned (Needleman-Wunsch algorithm; Needleman and Wunsch 1970) to derive a pairwise distance matrix, and the resultant matrix was clustered at a $98.5 \%$ similarity threshold to delineate haplotypes. Because of the high variability within the ITS2 region, this threshold allowed for calling haplotypes, while being conservative enough to not place all sequences into different haplotypes due to allelic contributions (Hughes et al. 2009).

Haplotype diversity within and among populations was evaluated using an analysis of molecular variance (AMOVA) in the program Arlequin (ver. 3.5.1.3; Excoffier and Lischer 2010). To discern biogeography of snow algae, a series of AMOVA models was used with samples grouped by (1) sampling location
(Colorado vs. Washington), (2) sampling year (2011 vs. 2012 in Washington only, as these were from the same glacial basin), and (3) Washington sites (the same locations were sampled in Washington for both 2011 and 2012). Models were run using 1000 permutations. Additionally, pairwise genetic distances for each alga between Washington samples for both 2011 and 2012 were generated between the dominant haplotype sequences using both uncorrected pairwise genetic distance (NeedlemanWunsch in Mothur) and corrected pairwise distance (Kimura two-parameter [K2P] distance in Arlequin). These pairwise distances were regressed with pairwise geographic distance to examine possible isolation by distance. To further interrogate isolation by distance, we performed correlation analyses between each algal pairwise geographic distance and genetic distance matrices using Mantel tests (1000 iterations). All statistical tests were done with a combination of Mothur, Arlequin, and JMP (ver. 7.0.2; SAS Institute, Cary, NC). Representative haplotype sequences were deposited at the National Center for Biotechnology Information under accession numbers KX063716KX063772, and all initial sequences are available as pairedend fastq in the Sequence Read Archive: SRR1104197 (BioProject accession number: PRJNA231162, BioSample accession number: SRS527350).

To confirm the putative taxonomic placement of our target algae, we conducted phylogenetic analyses. While the hypervariable ITS regions are not optimal for discerning higher-level phylogenetic relationships, they have great potential for resolving algal phylogenies at the species or genus levels (An et al. 1999). To generate maximum likelihood (ML) trees for confirmatory taxonomic placement, we used representative sequences of each delineated haplotype along with sequences deposited by the Culture Collection of Cryophilic Algae (CCCryo, www.cccryo .fraunhofer.de) that contained the full-length ITS2 region from GenBank. For Coenochloris trees, we included all CCCryo sequences and one full-length ITS2 sequence for a freshwater lake Coenochloris as well as all full-length ITS2 sequences from the closely related genus Sphaetocystis. Currently, the relationship between Coenochloris and Sphaetocystis is unresolved as taxa are often moved between these genera. For genus Chlamydomonas, we harvested all full-length ITS2 sequences deposited by the CCCryo as well as two sequences from the closely relatedgenus Chloromonas, whose placement is unresolved. Additional CCCryo-generated Chlamydomonadaceae clones were included. FASTA-formatted files of haplotype and reference sequences are provided in the supplemental files. These reference sequences were hand-edited to include only the ITS2 regions. Using the program Geneious (ver. 5.3.4; Biomatters, Auckland), representative haplotype sequences and reference ITS2 sequences were MAFFT aligned, and a maximum likelihood tree (PHYML) was generated using 100 iterations for each of the two snow algae.

Additionally, to determine whether these algae are phylogenetically clustered across a continental scale (Colorado vs. Washington), a neighbor-joining tree was generated (Geneious, using a Jukes-Cantor distance matrix) using 100 iterations employing all of the 800 sequences for each alga. To test whether these consensus trees were clustered differently than expected by random, the trees were analyzed using UniFrac (Lozupone and Knight 2005) using 1000 iterations with both weighted and unweighted options. 


\section{Results}

\section{Snow Algae Diversity}

The snow algae communities observed here include representatives from three orders and indicate taxonomically diverse snow algae communities comprised of few species. In all, there were 15 algal OTUs, the two most abundant of which were used for population analyses (Coenochloris sp. with best BLASTn match to accession HQ404874.1 [query coverage = $100 \%$, max identity $=82 \%$ ] and Chlamydomonas sp. with best BLASTn match to accession HQ404867.1 [query coverage $=100 \%$, max identity $=86 \%$ ]). Of the 15 OTUs (fig. 1) presented here, 11 are within the order Chlamydomonadales and likely represent species of Chlamydomonas and Chloromonas commonly observed in microscopic surveys of snow algae. However, because we exclusively queried these communities via sequencing and because of the poor representation of these species in the combined global nucleotide databases, confirming these taxonomic affinities is difficult. Additional taxa included three in the order Microthamniales (genus Trebouxia) and one in the order Chlorellales (genus Coenochloris). The OTU identified as Coenochloris sp. was most abundant by sequence count and over sevenfold more abundant than the next most abundant OTU.

\section{Haplotype Identification}

Using a $98.5 \%$ similarity threshold, the 800 Chlamydomonas sequences were distributed among 27 haplotypes and the 800 Coenochloris sequences among 30 haplotypes (see table A2 for haplotype identification). The algae present within each discrete patch belonged, with only one exception, to nonoverlapping haplotypes. We observed a complete independence of haplotype assignment across sites and years, even within geographically close sites between 2011 and 2012. Further, each algal patch was dominated by only one haplotype (sometimes exclusively), with a very minor proportion of subdominant haplotypes. In only one sampling location (Indian Peaks, 2012 , site 2 ) the dominant haplotype comprised a proportion $<80 \%$ : $54 \%$ of the sequences belonged to the dominant haplotype and $46 \%$ to a subdominant haplotype.

\section{Population-Level Haplotype Diversity}

Results of AMOVAs indicate that the majority of haplotype genetic diversity is explained by the among-population, withingroup variance component $\left(\Phi_{\mathrm{SC}}\right)$. This component explains at least $86 \%$ of the genetic variation, no matter what the group delineation (table 2). Interestingly, the within-population components are highly significant $\left(\Phi_{\mathrm{ST}}\right)$, even though this component represented only a small proportion of the total variation. Somewhat surprisingly, the among-group variance components $\left(\Phi_{\mathrm{CT}}\right)$ were negligible. This is particularly interesting given that our UniFrac analyses indicated strong terminal node clustering between Colorado and Washington locations. Both Chlamydomonas and Coenochloris were geographically isolated, as evidenced by significant UniFrac scores $(P<0.01$ for both alga $)$ between Washington and Colorado sites using either weighted or unweighted analyses after Bonferroni correction for multiple comparisons.

\section{Geographic Relationships}

Chlamydomonas sp. and Coenochloris sp. differed in their relationships between pairwise genetic and geographic distances. Uncorrected genetic distances show that the Coenochloris populations are strongly and positively correlated with geographic distances at the Washington sites in both $2011\left(R^{2}=0.443\right.$, $t=3.22, P=0.0067$; fig. $2 a)$ and $2012\left(R^{2}=0.460, t=\right.$ $3.33, P=0.0054$; fig. $2 b$ ). In contrast, genetic and geographic distances for Chlamydomonas populations showed no positive correlations in either $2011\left(R^{2}=0.016, t=-0.54, P=\right.$ 0.6015; fig. $2 c)$ or $2012\left(R^{2}=0.004, t=0.22, P=0.8325\right.$; fig. $2 d$ ). Mantel tests on matrix correlations corroborate our regression analyses. Coenochloris pairwise geographic and genetic matrices were positively correlated for both $2011(r=0.666$, $P=0.013)$ and $2012(r=0.678, P=0.021)$, whereas those of Chlamydomonas were correlated in neither $2011(r=$ $-0.147, P=0.624)$ nor $2012(r=0.060, P=0.434)$. When using the corrected genetic distance (K2P distance; see fig. A1; figs. A1-A3 available online), the differences between the two algae were similar but not as strong. It should be noted that the use of $\mathrm{K} 2 \mathrm{P}$ to generate genetic distances might not provide the best data to investigate DNA bar-code sequences (Collins et al. 2012; Collins and Cruickshank 2013) such as those analyzed here.

\section{Phylogenetic Analyses}

The confirmatory ML analyses support the BLASTn-based identification of these two algae OTUs to Chlamydomonas and Coenochloris. Snow Chlamydomonas haplotypes belong to a clade with Chlamydomonas sp. and Chlamydomonadaceae sp. references but not Chloromonas, another closely related genus often found in snow (fig. A2).

Coenochloris haplotypes are closely related to previously accessioned Coenochloris sequences (fig. A3). However, our environmental Coenochloris sequences are more closely relatednot to snow-borne Coenochloris sequences from CCCryo (primarily from Svalbard, Norway) but rather to the only other ITS2 Coenochloris sequence in GenBank (a freshwater isolate from Portugal).

\section{Discussion}

We present a first-of-its-kind analysis of the genetic and geographic distribution of two algae that dominate in snow algal communities. Similarly to other studies (Fujii et al. 2010; Remias et al. 2010), our data indicate a diverse algal community even though the snows seem to be visually dominated by a single taxon. The algae co-occur in great numbers within these discrete

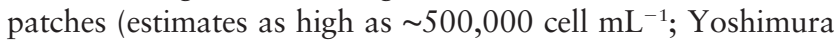
et al. 1997) but are rare in adjacent uncolonized snows (Brown et al. 2015a). The microhabitat attributes that allow for the establishment of these discrete algal patches are not yet understood. It is possible that establishment is stochastic and opportunistic. 
Table 2

Results of Analysis of Molecular Variance ( $\Phi$ Statistic) for Three Different Grouping Models

\begin{tabular}{|c|c|c|c|c|c|c|}
\hline \multirow[b]{2}{*}{ Structure } & \multicolumn{2}{|c|}{ WA/CO } & \multicolumn{2}{|c|}{ WA 2011/WA 2012} & \multicolumn{2}{|c|}{ WA sampling sites } \\
\hline & Coenochloris & Chlamydomonas sp. & Coenochloris & Chlamydomonas sp. & Coenochloris & Chlamydomonas sp. \\
\hline$\Phi_{\mathrm{ST}}$ & $.90376 * *(9.62)$ & $.86399 * * *(13.60)$ & $.94482 * * *(5.32)$ & $.88298 * *(11.70)$ & $.94409 * * *(5.59)$ & $.88275^{* * *}(11.73)$ \\
\hline$\Phi_{\mathrm{SC}}$ & $.90726 * *(94.15)$ & $.86610 * *(87.98)$ & $.94308 * * * 91.43)$ & $.88199 * *(87.46)$ & $.94349 * *(93.35)$ & $.88035 * *(86.27)$ \\
\hline$\Phi_{\mathrm{CT}}$ & $-.03776(-3.78)$ & $-.01579(-1.58)$ & $.03056(3.06)$ & $.00842(.84)$ & $.01061(1.06)$ & $.02007(2.01)$ \\
\hline
\end{tabular}

Note. $\quad \Phi_{\mathrm{ST}}=$ within-population variance; $\Phi_{\mathrm{SC}}=$ among-population, within-groups variance; $\Phi_{\mathrm{CT}}=$ among-groups variance; WA/CO $=$ populations in Washington $(n=12)$ versus Colorado $(n=4)$, combined across years (2011 and 2012); WA 2011/WA $2012=$ Washington-only samples for $2011(n=6)$ and $2012(n=6)$; WA sampling sites $=$ the same sampling locations combined across years $(2011$ and 2012) as outlined in table 1 . Shown are the $\Phi$ statistics and the percentage of variation explained within each model (in parentheses).

$\because * P<0.001$.

It may be that once an algal propagule successfully establishes under favorable conditions or in the presence of available nutrients (most likely nitrogen, which may be deposited atmospherically or via fecal droppings from migratory birds), it reproduces asexually in great numbers. This establishment may be aided by diazotrophs that initialize a positive feedback loop, further enriching the patch with nitrogen and carbon (Gosselin et al. 1990). The nutrient inputs may further facilitate the estab- lishment and population growth of other algae and microbes, including bacteria and fungi that may be enriched within the algal patch (Brown et al. 2015a). The founder effects from few propagules and intense kin competition potentially explains why only few haplotypes occur and dominate each patch.

One of the more interesting findings is the extreme site specificity of the haplotypes (see table A2; figs. A2, A3). Both Coenochloris sp. and Chlamydomonas sp. within the algal patches
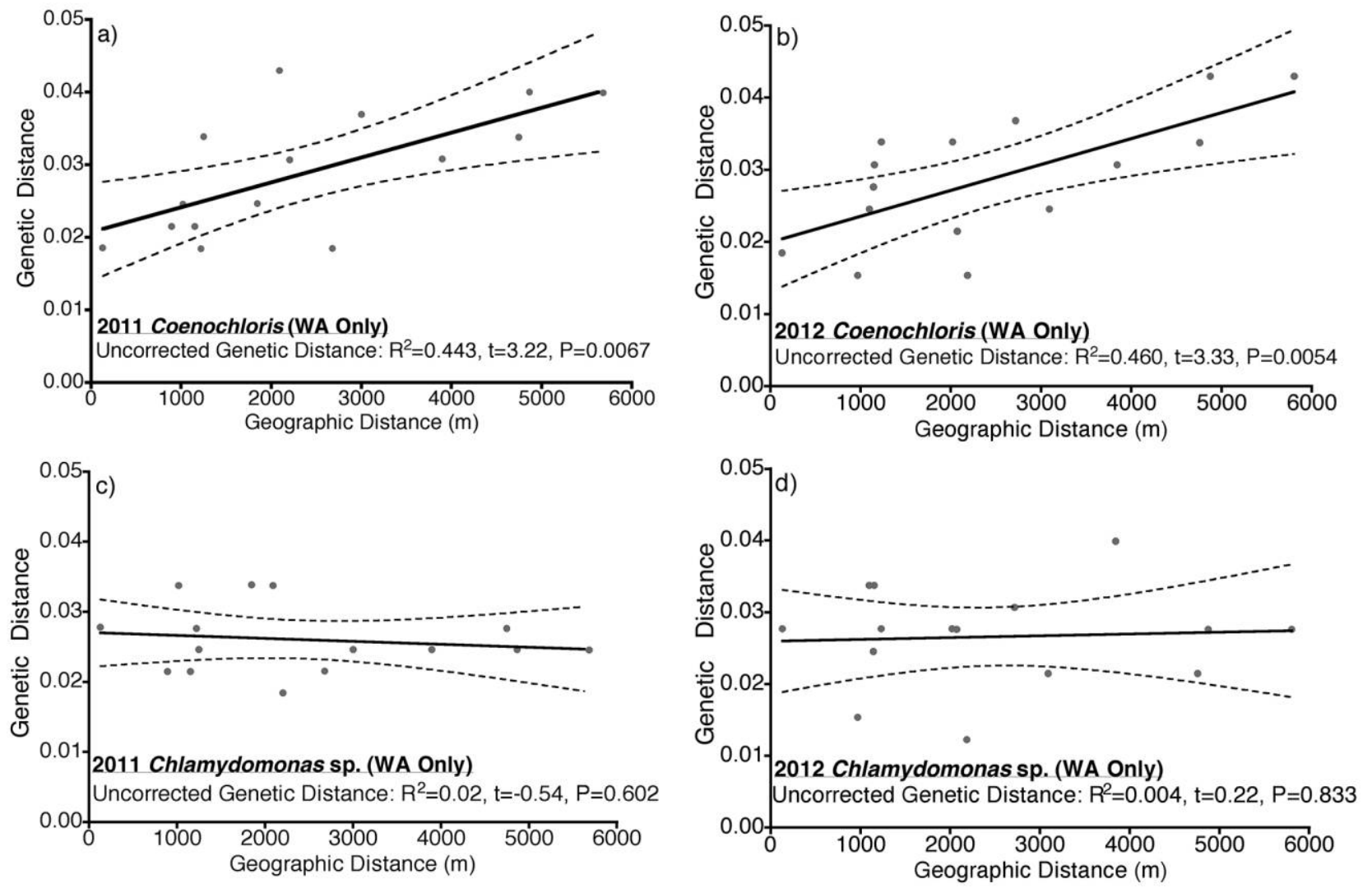

Fig. 2 Regression between the sampling location geographic and haplotype genetic distances. Note that regression analyses of Coenochloris for $2011(a)$ and $2012(b)$ indicate a positive relationship between geographic and genetic distances indicative of biogeographic structuring. In contrast, similar regression analyses of Chlamydomonas sp. indicated no geographic structuring either in 2011 (c) or 2012 (d) sampling. 
are strongly dominated by one haplotype, with few and often no subdominant representatives. The lack of subdominant haplotypes may be a result of our shallow sampling (50 sequences per algae patch per species), and deeper interrogation would likely show more haplotype diversity; however, our Boneh estimates suggest that this is unlikely. Further, any additional subdominant haplotypes that may be gained would likely not have proportions greater than the $\sim 2 \%$ seen here. The subdominant haplotypes are, with few exceptions, very closely related to the dominant haplotype in our ML analyses. Our data suggest that while the snow algal patches are comprised of multiple species, each species within a patch is highly clonal. It is likely that the observed clonality is primarily driven by strong priority effects that inhibit successful establishment of subsequently arriving propagules and strong kin competition that structure algal communities at small local scales, that is, within each colonized patch. This may also be a result of either low propagule inputs and/or viabilities combined with stochastic establishment, or it may be a result of reestablishment of algae in situ from the previous growing season that controls the population/community assembly within a colonized patch.

The diploid zygotic stage of these algae dominates the algal blooms in snows (Hoham et al. 1983). These zygotes likely propagate asexually, locally producing clonal populations in extremely large numbers. Our analyses indicate that the subdominant haplotypes within a patch are closely related to the dominant haplotype, potentially implying sexual recombination with close kin. Alternatively, although these algae are largely clonal, they may also reproduce unisexually, explaining the distinct but closely related subdominant haplotype occurrence. Given that haplotypes that occupy the same snow patch are very closely related, it is conceivable that these subdominant haplotypes arise from the parent population (dominant haplotype) of the major haplotype due to mutations (or unisexual reproduction). For the $325 \mathrm{bp}$ sequences queried here, only five nucleotide differences are required to be distinct for inclusion into a new haplotype. These newly mutated, closely related haplotypes are then subjected to very strong kin competition that continues to suppress the subdominant haplotype frequencies. The lack of geographic structuring suggests that these algae can disperse to cross with other patches. However, our data do not permit elucidating the reproductive strategies or the genesis of subdominant haplotypes; further investigation is required to adequately address these questions.

Our data also suggest contrasting life-history strategies of Coenochloris and Chlamydomonas. In our analyses, Coenochloris are strongly geographically structured, as evidenced by the positive correlations between genetic and geographic distances (fig. $2 a$, 2b), whereas Chlamydomonas are not. Our data suggest some isolation by distance in Coenochloris. Such isolation may prevent crosspatch dispersal and suggests rampant near-neighbor mating with little long-distance propagule dispersal for reproduction. In contrast, snow-borne Chlamydomonas seem read- ily dispersed to regionally discrete patches during the reproductive cycle, as our data provide no evidence for geographic structure in the Chlamydomonas populations. This suggests that Chlamydomonas gametes may be readily dispersed across the landscape.

The contrasting patterns of isolation by distance may suggest different dispersal capabilities of these two algae, likely driven by propagating unit size. The average spherical zygote of Chlamydomonas nivalis is $14.9 \mu \mathrm{m}$ in diameter (Remias et al. 2005), whereas the propagating unit (encasement of up to 16 zygotes in a mucilaginous envelope) of Coenochloris sp. may be up to $60 \mu \mathrm{m}$ in diameter (Fott 1974). Chlamydomonas sp. is not dispersal limited on a regional scale, as evidenced by the lack of any correlation between the genetic and geographic distances across Washington's Lyman Glacier basin in either 2011 or 2012. This indicates that Chlamydomonas sp. is readily transported on regional scales. In contrast, Coenochloris sp. populations were structured geographically, and their genetic distances tended to be correlated with geographical distance. The haplotype-specific patches support aerial dispersal in short distances. However, the mechanisms and the potential for longdistance propagule dispersal remain uncertain.

Our study demonstrates that taxonomically broad multispecies algal communities with few members colonize discrete snow patches. While comprised of a few species, and thus possessing interspecific variability, each species within a snow patch seems to be highly clonal and with only little intraspecific variability. Although our analyses indicated that both targeted taxa possessed similarly low intraspecific variability, their populations on larger, regional scales were differently structured. These observations suggested contrasting dispersal capabilities. Snow algae are communities of clones, and these clonal communities are likely structured via founder or priority effects and intense kin competition as well as dispersal limitations. These attributes make snow algae an exceptional study system and one uniquely suited to test hypotheses of organismal persistence and ecological selection pressures.

\section{Acknowledgments}

We wish to acknowledge Leah Fitzwater for assistance with DNA extractions and Vera Brown, Ashlyn Jumpponen, Kale Lothamer, and Corin White for assistance with field collections. We wish to express thanks to all the anonymous people who helped S. P. Brown after a field injury. Kale Lothamer was supported by Undergraduate Research Mentoring in the Ecological Genomics program (National Science Foundation [NSF] grant 1041199). S. P. Brown was supported by the Graduate Awards in Areas of National Need program from the Department of Education and GK-12 (NSF DGE-0841414) awards. This work was partially funded by a Kansas Academy of Science graduate research grant to S. P. Brown.

\section{Literature Cited}

An SS, T Friedl, E Hegewald 1999 Phylogenetic relationships of Scenedesmus and Scenedesmus-like coccoid green algae as inferred from ITS-2 rDNA sequence comparisons. Plant Biol 1:418-428.
Boneh A, A Boneh, RJ Caron 1998 Estimating the prediction function and the number of unseen species in sampling with replacement. I Am Stat Assoc 93:372-379. 
Brown SP, BJSC Olson, A Jumpponen 2015a Fungi and algae cooccur in snow: an issue of shared habitat or algal facilitation of heterotrophs? Arct Antarct Alp Res 47:727-747.

Brown SP, AM Veach, AR Rigdon-Huss, K Grond, SK Lickteig, K Lothamer, AK Oliver, A Jumpponen $2015 b$ Scraping the bottom of the barrel: are rare high throughput sequences artifacts? Fungal Ecol 13:221-225.

Collins RA, LM Boykin, RH Cruichshank, KF Armstrong 2012 Barcoding's next top model: an evaluation of nucleotide substitution models for specimen identification. Methods Ecol Evol 3:457-465.

Collins RA, RH Cruickshank 2013 The seven deadly sins of DNA barcoding. Mol Ecol Resour 13:969-975.

De Clerck O, MD Guiry, F Leliaert, Y Samyn, H Verbruggen 2013 Algae taxonomy: a road to nowhere? I Phycol 49:215-225.

Excoffier L, HEL Lischer 2010 Arlequin suite ver 3.5: a new series of programs to perform population genetics analyses under Linux and Windows. Mol Ecol Resour 10:564-567.

Fogg GE 1967 Observations on the snow algae of the South Orkney Islands. Philos Trans R Soc B 252:279-287.

Fott B 1974 Taxonomie der palmelloiden Chlorococcales (Familie Palmogloeaceae). Preslia 46:1-31.

Fujii M, Y Takano, H Kojima, T Hoshino, R Tanaka, M Fukui 2010 Microbial community structure, pigment composition, and nitrogen source of red snow in Antarctica. Microbial Ecol 59:466-475.

Gorton HL, WE William, TC Vogelmann 2001 The light environment and cellular optics of the snow alga Chlamydomonas nivalis (Bauer) Wille. Photochem Photobiol 73:611-620.

Gosselin M, L Legendre, J Therriault, S Demers 1990 Light and nutrient limitations of sea-ice microalgae (Hudson Bay, Canadian Arctic). I Phycol 26:220-232.

Gradinger R, D Nürnberg 1996 Snow algae communities on Arctic pack ice floes dominated by Chlamydomonas nivalis (Bauer) Wille. Proc NIPR Symp Polar Biol 9:35-43.

Grinde B 1983 Vertical distribution of the snow alga Chlamydomonas nivalis (Chlorophyta, Volvocales). Polar Biol 2:159-162.

Hoham RW, B Duval 2001 Microbial ecology of snow and freshwater ice with emphasis on snow algae. Pages 186-203 in HG Jones, HG Pomeroy, DA Walker, RW Hoham, eds. Snow ecology: an interdisciplinary examination of snow-covered ecosystems. Cambridge University Press, Cambridge.

Hoham RW, JE Mullet, SC Roemer 1983 The life history and ecology of the snow alga Chloromonas polyptera comb nov. (Chlorophyta, Volvocales). Can I Bot 61:2416-2429.

Hughes KW, RH Petersen, EB Lickey 2009 Using heterozygosity to estimate a percentage DNA sequence similarity for environmental species' delimitation across basidiomycete fungi. New Phytol 182: 795-798.

Ihrmark K, ITM Bödeker, K Cruz-Martinez, H Friberg, A Kubartova, J Schenck Y Strid, et al 2012 New primers to amplify the fungal ITS2 region-evaluation by 454-sequencing of artificial and natural communities. FEMS Microbiol Ecol 82:666-677.

Lozupone C, R Knight R 2005 UniFrac: a new phylogenetic method for comparing microbial communities. Appl Environ Microbiol 71: $8228-8235$.

Lutz S, AM Anesio, A Edwards, LG Benning 2015 Microbial diversity on Icelandic glaciers and ice caps. Front Microbiol, doi:10.3389 /fmicb.2015.00307.

Müller T, W Bleiß, CD Martin, S Rogaschewski, G Fuhr 1998 Snow algae from northwest Svalbard: their identification, distribution, pigment and nutrient content. Polar Biol 20:14-32.

Needleman SB, CD Wunsch 1970 A general method applicable to the search for similarities in the amino acid sequences of two proteins. I Mol Biol 48:443-453.

Remias D, U Karsten, C Lütz, T Leya 2010 Physiological and morphological processes in the alpine snow alga Chloromonas nivalis (Chlorophyceae) during cyst formation. Protoplasma 242:73-86.

Remias D, U Lütz-Meindl, C Lütz 2005 Photosynthesis, pigments and ultrastructure of the alpine snow alga Chlamydomonas nivalis. Eur I Phycol 40:259-268.

Schloss PD, SL Westcott, T Ryabin, JR Hall, M Hartmann, EB Hollister, RA Lesniewski, et al 2009 Introducing mothur: opensource, platform-independent, community-supported software for describing and comparing microbial communities. Appl Environ Microbiol 75:7537-7541.

Takeuchi N 2001 The altitudinal distribution of snow algae on an Alaska glacier (Gulkana Glacier in the Alaska Range). Hydrolog Process 15:3447-3459.

2013 Seasonal and altitudinal variations in snow algae communities on an Alaskan glacier (Gulkana glacier in the Alaska range). Environ Res Lett 8:035002.

Takeuchi N, S Kohshima 2004 A snow algal community on Tyndall Glacier in the Southern Patagonia Icefield, Chile. Arct Antarct Alp Res 36:92-99.

White TJ, T Bruns, S Lee, JW Taylor 1990 Amplification and direct sequencing of fungal ribosomal RNA genes for phylogenetics. Pages 315322 in MA Innis, MA Gelfand, JJ Sininsky, TF White, eds. PCR protocols: a guide to methods and applications. Academic Press, New York.

Yoshimura Y, S Kohshima, S Ohtani 1997 A community of snow algae on a Himalayan glacier: change of algal biomass and community structure with altitude. Arct Alp Res 29:126-137. 